\title{
NEUTRINO OSCILLATIONS AT REACTORS: WHAT NEXT?
}

\author{
L.A. Mikaelyan, V.V. Sinev \\ RRC "Kurchatov Institute", Kurchatov Sq., 1, Moscow-123182, Russia. \\ Talk given at the International Conference on Non-Accelerator New Physics, \\ NANP'99, Dubna, 28.06-3.07. 1999. \\ We shortly review past and future experiments at reactors aimed at searches \\ for neutrino masses and mixing. We also consider new idea to search at \\ Krasnoyarsk for small mixing angle oscillations in the atmosheric neutrino \\ mass parameter region.
}

\section{Introduction}

The first long baseline reactor experiment CHOOZ'97 [1] has successfully reached the atmospheric mass parameter region $\delta m_{\text {atm }}^{2} \sim 10^{-3} \mathrm{eV}^{2}$ and has tested there a large portion of the area of interest on the $\delta m^{2}-\sin ^{2} 2 \theta$ plane. No signs of oscillations have been found. Thus oscillations of electron neutrinos can NOT dominate in the atmospheric neutrino anomaly.

The Super-Kamiokande data on atmospheric neutrinos provide strong evidence in favor of intensive $\nu_{\mu} \rightarrow \nu_{X} \quad(x \neq e)$ transitions [2]. In the three active neutrino $\left(\nu_{e}, \nu_{\mu}, \nu_{\mu}\right)$ oscillation model considered here $\nu_{X}=\nu_{\tau}$.

We wish to emphasize however that both experiments, CHOOZ'97 and SK, do not exclude $\nu_{e} \leftrightarrow \nu_{\mu}$ oscillations as a subdominant mode in the $\delta m_{a t m}^{2}$ region $[3,4]$.

The results of recent experiments have attached great attention to the problem of neutrino oscillations. New physical ideas and projects of new large scale experiments at accellarators are actively discussed (for review cf. Ref.[4]).

What new contributions can be done with reactor $\widetilde{\nu}_{e}, e$ 's for exploring the electron neutrino mass and mixing problems? 
One line of future studies has already been announced. To probe the large mixing angle (LMA) MSW solution $\left(\delta m_{\text {sol }}^{2} \simeq 10^{-4}-10^{-5} \mathrm{eV}^{2}\right.$, $\left.\sin ^{2} 2 \theta \sim 0.7\right)[5]$ of the solar neutrino puzzle the projects KamLAND at Kamioka [6] and BOREXINO at Gran Sasso [7] plan to detect neutrinos from reactors which operate hundred kilometers away from the detector sites.

In this report we consider another possibility. We find that with two detector techniques the sensitivity to the mixing parameter in the $\delta m_{\text {atm }}^{2}$ region can be substantially increased in comparison with that achieved in CHOOZ experiment. We suggest a new study of the problem at Krasnoyarsk underground (600 m.w.e.) laboratory with detectors stationed at $1100 \mathrm{~m}$ and $250 \mathrm{~m}$ from the reactor. The main goals of the proposed experiment are:

- To obtain better understanding of the role the electron neutrino can play in the atmospheric neutrino anomaly,

- To obtain new information on neutrino mixing, here the $U_{e 3}$ element of the neutrino mixing matrix,

- To give normalization for future long baseline experiments at accellarators.

\section{OSCILLATIONS OF REACTOR ANTI- NEUTRINOS}

Nuclear reactor generates antineutrinos at a rate $N_{\nu} \sim 1.8 \cdot 10^{20} s^{-1}$ per $1 \mathrm{GWt}$ thermal power. The typical reactor $\widetilde{\nu}_{e}$ energy spectrum normalized to one fission event is presented in Fig.1.

The $\widetilde{\nu}_{e} \mathrm{~s}$ are detected via the inverse beta decay reaction

$$
\widetilde{\nu}_{e}+p \rightarrow e^{+}+n,
$$

The positron kinetic energy $\mathrm{T}$ is related to the energy $\mathrm{E}$ of the $\widetilde{\nu}_{e}$ as

$$
T=E-1.804 \quad M e V .
$$

The signature that identifies the $\widetilde{\nu}_{e}$ absorption in the liquid scintillator target is a spatially correlated delayed coincidence between the prompt positron and the signal from the neutron capture $\gamma$-rays. 
The probability $\mathrm{P}\left(\widetilde{\nu}_{e} \rightarrow \widetilde{\nu}_{e}\right)$ for $\widetilde{\nu}_{e}$ to survive at the distance of $\mathrm{R}$ meters from the source is given by the expression

$$
P\left(\widetilde{\nu}_{e} \rightarrow \widetilde{\nu}_{e}\right)=1-\sin ^{2} 2 \theta \cdot \sin ^{2}\left(1.27 \delta m^{2} \cdot R \cdot E^{-1}\right),
$$

$\mathrm{E}(\mathrm{MeV})$ is the neutrino energy, $\delta m^{2}$ is the mass parameter in $e V^{2}$ and $\sin ^{2} 2 \theta$ is the mixing parameter.

The distortion of the positron energy spectrum and the deficite of the total $\widetilde{\nu}_{e}$ detection rate relative to no-oscillation case are the signatures for oscillations that are searched for in the experiment. The deficite of the total rate is the strongest for $\left(R \cdot \delta m^{2}\right)_{\max } \approx 5 \mathrm{~m} \cdot \mathrm{eV}^{2}$.

In the pressurized water reactors (PWR) $\widetilde{\nu}_{e}$ spectrum and total cross section of the reaction (1) vary with the nuclear fuel composition, (the burn up effect). The current fuel composition is provided by reactor services. When the fuel composition is known the no-oscillation cross section $\sigma_{V-A}$ can be calculated within the uncertainty of $2.7 \%$. (For more information see e.g.[8] and references therein).

With the help of an integral type detector the CdF-KURCHATOVLAPP group has accurately measured the cross section at a $15 \mathrm{~m}$ distance from the Bugey-5 reactor [9]:

$$
\sigma_{\text {exper }}=5.750 \cdot 10^{-43} \mathrm{~cm}^{2} / \text { fission } \pm 1.4 \%
$$

This highly accurate value $\sigma_{\text {exper }}$ can be used in other experiments with reactor $\widetilde{\nu}_{e}$ 's as a no-oscillation metrological standard. When it is used in practice one must consider the differences in the fuel compositions and take into account a number of "small effects". This increases the error up to about $2 \%$.

\section{PAST, CURRENT AND FUTURE EXPERIMENTS}

During 1980 - 1995 yy. intensive searches for neutrino oscillations with detectors located at $\sim 10-230 \mathrm{~m}$ from relevant reactors have been performed. These "short baseline" experiments are listed in the left part of Fig.2. The highest sensitivity to the mixing parameter $\left(\sin ^{2} 2 \theta \approx 0.02\right)$ was reached by the Bugey-3 group in the measurements with two iden- 
tical detectors located at $15 \mathrm{~m}$ and $40 \mathrm{~m}$ from the reactor [9] (see Fig.3).

The CHOOZ detector used a 5 tons liquid scintillator (Gd) target. It was located in an underground laboratory (300 MWE) at a distance of about $1 \mathrm{~km}$ from the neutrino source. The ratio $\mathrm{R}$ of the measured to expected in no oscillation case neutrino detection rates was

$$
R=0.98+0.04(\text { stat })+0.04(\text { syst }), / \text { November } 1997 / .
$$

The systematic errors come mainly from the reactor properties and the absolute values of neutrino detection efficiencies. The 90\% CL exclusion plot "CHOOZ'97" for $\widetilde{\nu}_{e}$ disappearance channel is presented in Fig.3. together with the allowed $\nu_{\mu} \rightarrow \nu_{\tau}$ oscillation channel SK'736 d [2] (shaded area). The experiment was continued till June 1998 y. to achieve better statistics and improve systematics. The final CHOOZ results will appear soon.

The Palo Verde oscillation experiment at $\sim 800 \mathrm{~m}$ from three reactors is taking data since October 1998. The first 70 day results are now available [11].

Past and current experiments cover now the distances up to $1 \mathrm{~km}$ from the reactor. The extension to $\sim 200$ and $\sim 800 \mathrm{~km}$ is expected with the forthcoming KamLAND and BOREXINO ultra long baseline projects (Fig.2). They will use liquid scintillator targets of 1000 and 300 tons respectively. The solar large mixing angle MSW solution [7] is well inside the area planned for the investigation (Fig.3).

The experimental goal of the new search at Krasnoyarsk is to extend studies to the "white spot" area left to the CHOOZ limits in Fig.3.

\section{NEW PROJECT FOR KRASNOYARSK}

\subsection{Detectors}

Two identical liquid scintillation spectrometers stationed at the Krasnoyarsk underground site (600 MWE) at distances $R_{1}=1100 \mathrm{~m}$ and $R_{2}$ $=250 \mathrm{~m}$ from the reactor source simultaneously detect $\left(e^{+}, \mathrm{n}\right)$ pairs produced via reaction (1). A simplified version of the BOREXINO detector composition is chosen for the design of the spectrometers (Fig.4). The 50 ton targets in the center of the detectors (mineral oil + PPO) 
Table 1: Antineutrino detection rates $N\left(e^{+}, \mathrm{n}\right) d^{-1}$.

\begin{tabular}{|c|c|cc|c|c|}
\hline Detector & CHOOZ'97 & THIS PROJECT & KamLand & BOREXINO \\
\hline $\begin{array}{c}\text { Mass of the } \\
\text { target, tons }\end{array}$ & 5 & 50 & 50 & 1000 & 300 \\
$\begin{array}{c}\text { Distance from } \\
\text { the source, km }\end{array}$ & 1 & 0.25 & 1.1 & $\sim 200$ & $\sim 800$ \\
$N\left(e^{+}, \mathrm{n}\right) d^{-1}$ & 12 & 1000 & 55 & 2 & 0.08 \\
\hline
\end{tabular}

are viewed by the PMT's $(\sim 20 \%$ coverage, $\sim 120$ ph.e. $/ \mathrm{MeV})$ through $\sim 1 \mathrm{~m}$ thick layer of non-scintillating oil.

Computed neutrino detection rates can be seen in the middle of Table 1. For comparison parameters of the CHOOZ and future KamLAND and BOREXINO detectors is also included.

\subsection{Backgrounds}

The CHOOZ experiment has demonstrated revolutionary improvements of the reactor neutrino techniques: a 500 - 1000 times lower level the background has been achieved relative to previous experiments at reactors (see the first three colomns in Tab.2).

It is important to note that with the CHOOZ experience and also with detailed studies performed at the BOREXINO CTF detector [7] the main secrets of the background supression are now well understood, at least for the level we need here. The secrets are:

- Detector should be stationed sufficiently deep underground to reduce the the flux of cosmic muons, the main source of the background in these type experiments.

- To reduce the accidental background the potomultiplyers with their highly radioactive glass should be separated from the central scintillator volume by sufficiently thick layer of the oil ("Borexino geometry", Fig.4). 
Table 2: Neutrino signal $N\left(e^{+}, \mathrm{n}\right)$ and background $N_{B K G}$ rates (per 1 day, per 1 ton of scintillator target).

\begin{tabular}{|c|c|c|c|c|c|c|}
\hline Detector & Rovno & Bugey $^{*}$ & CHOOZ'97 $^{\text {This }}$ & $\begin{array}{c}\text { Kam } \\
\text { Project }^{* *}\end{array}$ & BOREXINO & \\
\hline$M W E^{* * *}$ & 30 & $\sim 10$ & 300 & 600 & 2700 & 3200 \\
$N\left(e^{+}, \mathrm{n}\right)$ & 1700 & 370 & 2.4 & 1.1 & $2 \cdot 10^{-3}$ & $3 \cdot 10^{-4}$ \\
$N_{B K G}$ & 220 & 160 & 0.24 & $\sim 0.1$ & $<10$ & $<10$ \\
\hline
\end{tabular}

* Detector at $40 \mathrm{~m}$ position.

** Detector at $1100 \mathrm{~m}$ position.

*** Overburden in meters of water equivalent.

We estimate the total background rate as 0.1 per day, per ton of the target. It is 2.5 times lower than the background measured at CHOOZ, which seems reasonable for a detector located twice as deep underground (compare Tab.2).

\subsection{Data analysis}

In three years of data taking $40 \cdot 10^{3}$ neutrino events with 10:1 the signal to background ratio and about $800 \cdot 10^{3}$ events can be accumulated at the $1100 \mathrm{~m} 250 \mathrm{~m}$ positions respectively.

Two types of analysis can be used. Both of them are not affected by the value of the absolute $\widetilde{\nu}_{e}$ flux and $\widetilde{\nu}_{e}$ energy spectrum, reactor power, the burn up effects and absolute values of the detector efficiencies.

Analysis I is based on the ratio $X_{R A T E}=N_{1} / N_{2}$ of the neutrino detection rates measured at two distances:

$$
X_{R A T E}=\frac{R_{2}^{2} \cdot \epsilon_{1} \cdot V_{1}}{R_{1}^{2} \cdot \epsilon_{2} \cdot V_{2}} \cdot F\left(\delta m^{2}, \sin ^{2} 2 \theta\right)
$$

$\epsilon_{1,2}$ and $V_{1,2}$ are the neutrino detection efficiencies and the scintillator volumes.

Thus the absolute values of detection efficiencies are practically conceled, only their small relative differences are to be considered here. 
Analysys II is based on the comparison of the shapes of the $e^{+}$ spectra $S\left(E_{e}\right)$ measured simultaneously in two detectors. Small deviations of the raio $X_{S H A P E}=S_{1} / S_{2}$

$$
X_{S H A P E}=C \cdot\left(1-\sin ^{2} 2 \theta \sin ^{2} \phi_{1}\right) \cdot\left(1-\sin ^{2} 2 \theta \sin ^{2} \phi_{2}\right)^{-1},
$$

from a constant value are searched for the oscillation effects $\left(\phi_{1,2}\right.$ stands for $1.27 \delta m^{2} R_{1,2} E^{-1}$ ). No knowleadge of the constant $\mathrm{C}$ in Eq. (6) is needed for this analysis so that the details of the geometry, ratio of the target volumes and efficiencies are excluded from the consideration.

\subsection{Detector calibrations}

Calibrations of the detectors are of crucial importance. Difference between the respons functions of the two detectors which is difficult to avoid can produce some modulation of the ratio (6) and thus imitate the oscillation effect. The differences can be measured and relevant corrections found. This can be done by systematic intercomparison of the scales in many energy points using the sources of $\gamma$-rays shown in Fig.5.

An additional approach is also considerd. The spectrometers can be tested periodically with the use of the ${ }^{252} C f$ spontaneous fission source that can produce a broad spectrum due to prompt $\gamma$-rays and neutron recoils (Fig.5). The ratio of these spectra should be constant, if the instrumental modulation is observed it can be measured and used to find corrections to the Eq.(6).

\subsection{Expected constraints on oscillation parameters}

We hope that the ratio $\epsilon_{1} V_{1} / \epsilon_{2} V_{2} \approx 1$ (Eq.5) can be controlled within $0.8 \%$. Then from Analysis I we expect the 90\% CL limits shown in Fig.6 (the curve labelled "RATE").

We believe that the false effects in Eq.(6) can be controlled down to the level of $0.5 \%$. Relevant $90 \%$ CL limits are presented in Fig.6 (the curve "SHAPE"). 


\section{DISCUSSION AND CONCLUSIONS}

With methods of data analysis mentioned in Sec.4 we arrive at the limits on the oscillation parametes decoupled from the main sources of systematic uncertainties which limit the sensitivity of the experiments based on absolute comparison of the measured and expected no-oscillation rates and/or positron spectra.

Nevertheless the systematic errors which remain notably decrease the sensitivity to the mixing parameter $\sin ^{2} 2 \theta$. The curve "SHAPE" (Fig.6) is about two times less restrictive as compared to the statistical limits found for an ideal detector with no systematics.

Now we come back to the main question: what contributions to the neutrino physics can be expected from new oscillation experiments at reactors?

The long baseline (LBL) experiments with detectors stationed at a distance of $\sim 1 \mathrm{~km}$ from the reactor search for the mixing parameter $\sin ^{2} 2 \theta_{L B L}$ which is expressed in this case as

$$
\sin ^{2} 2 \theta_{L B L}=4 \cdot U_{e 3}^{2}\left(1-U_{e 3}^{2}\right),
$$

$U_{e 3}^{2}$ is the contribution of the heaviest mass eigenstate $\nu_{3}$ to the flavor electron neutrino state:

$$
\nu_{e}=U_{e 1} \nu_{1}+U_{e 2} \nu_{2}+U_{e 3} \nu_{3}
$$

From CHOOZ'97 results we already know that $U_{e 3}^{2}$ is not large: $U_{e 3}^{2}<$ $(3-5) \cdot 10^{-2}$. Future experiment at $1 \mathrm{~km}$ considerd here can find $U_{e 3}^{2}$ or set a much smaller upper limit. Therefore better understanding of the neutrino mixing can be achieved. New information on $U_{e 3}$ can be useful for analysis of the atmospheric neutrinos and can give hints for future long baseline experiments at accelerators.

The ultra long baseline (ULBL) experiments KamLAND and BOREXINO will search for $\sin ^{2} 2 \theta_{U L B L}$ which depends on the contributions of the $\nu_{1}$ and $\nu_{2}$ mass states:

$$
\sin ^{2} 2 \theta_{U L B L}=4 \cdot U_{e 1}^{2} U_{e 2}^{2}
$$

We conclude that the experiments at reactors discussed here can provide full information of the mass structure of the electron neutrino, at least in the three neutrino oscillation model. 


\section{Acknowledgments}

We greatly appreciate fruitful discussions with S.Bilenky, E. Lisi and A. Smirnov. We thank our colleagues V. Martemyanov, Yu. Kozlov and V. Vyrodov for many discussions. This study is supported by RFBR.

\section{References}

[1] M.Apollonio et al. Phys. Lett. B 420, 397 (1998);

CHOOZ Collab., hep-ex/9711002.

[2] Y.Fukida et al., Super-Kamiokande Coll., Phys. Rev. Lett. 81, N8, 1562 (1998);

K.Scholberg, Super-Kamiokande Coll., hep-ex/9905016.

[3] G.Fogli, E.Lisi, A.Marrone, G.Scioscia, hep-ph/9904465.

[4] S.Bilenky, C.Giunti, W.Grimus, in Prog. Part. Nuc. Phys., v.43, to be published; available at hep-ph/9812360.

[5] J.Bachall, P.Krastev, A.Smirnov, Phys. Rev. D58, 096016 (1998);

J.Bachall, P.Krastev, A.Smirnov, hep-ph/9905220.

[6] F.Suekane, Worksop NOW'98, 7-9 September 1998, Amsterdam, http://www.nikhef.nl/pub/conferences/now98;

G.Gratta, hep-ex/9905011.

[7] L.Oberauer, Borexino Coll., Neutrno'98, http://www-sk.icrr.u-tokyo.ac.jp/nu98;

http://almime.mi.infn.it/html/papers/index.html.

[8] V.Kopeikin, L.Mikaelyan, V.Sinev, Russ. Yad. Fiz. 60, 230 (1997);

Phys. At. Nucl. 60, 172 (1997).

[9] V.Vyrodov et al, JETP Lett. v.61, No 3, (1995);

Y.Declay et al., Phys. Lett. B 338, 383 (1994).

[10] B.Achkar et al. Nucl.Phys.B 434, 503 (1995)

[11] F.Boehm, Palo Verde, http://citnp.caltech.edu/PV/Palo-Verde/html. 


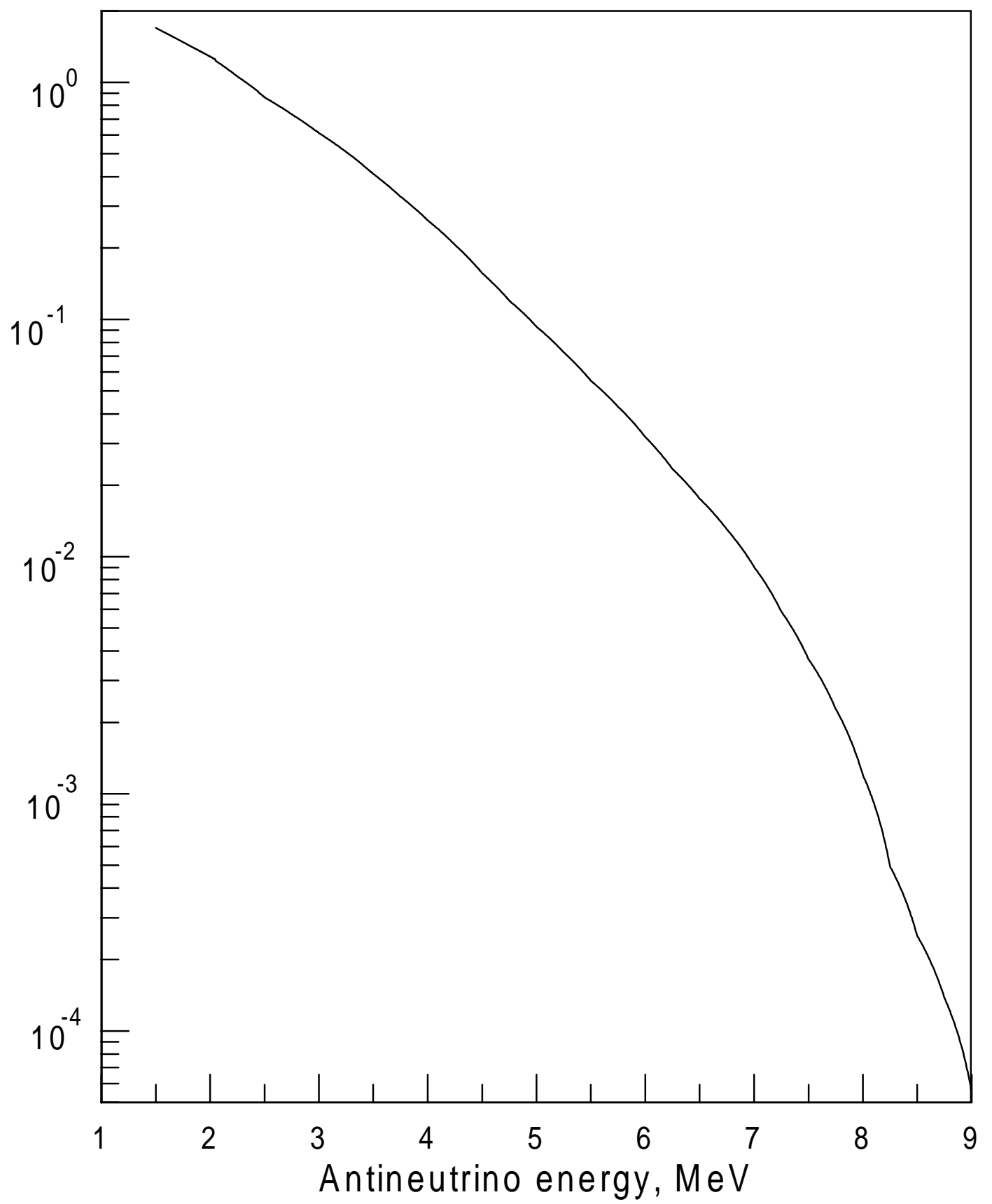

Figure 1. Energy spectrum of reactor antineutrinos. 
Distance from the reactor source

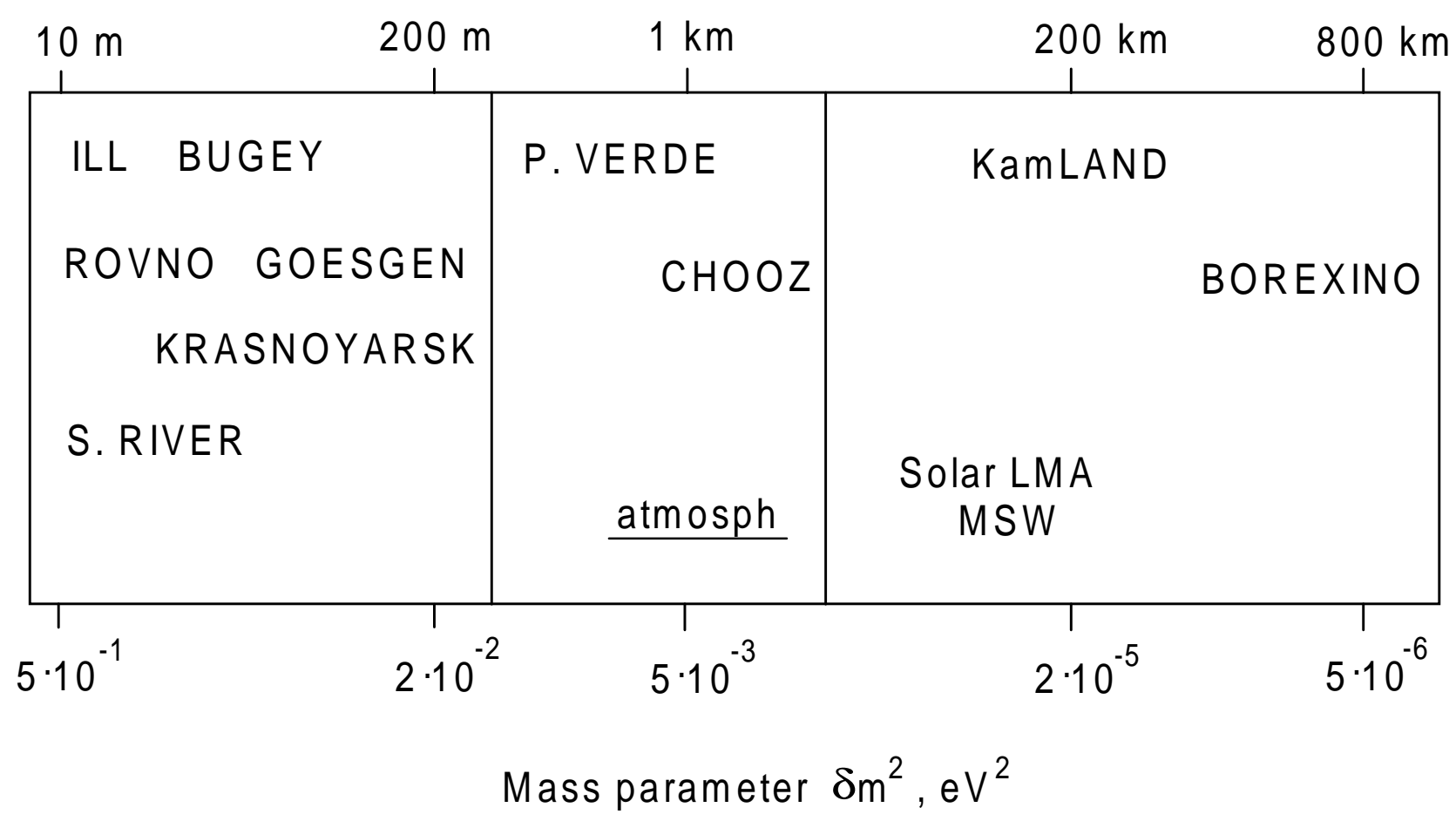

Figure 2. Reactor oscillation experiments.

Left: Past short baseline; Middle: Current long baseline; Right: Future ultra long baseline. New Krasnoyarsk project is not included. 


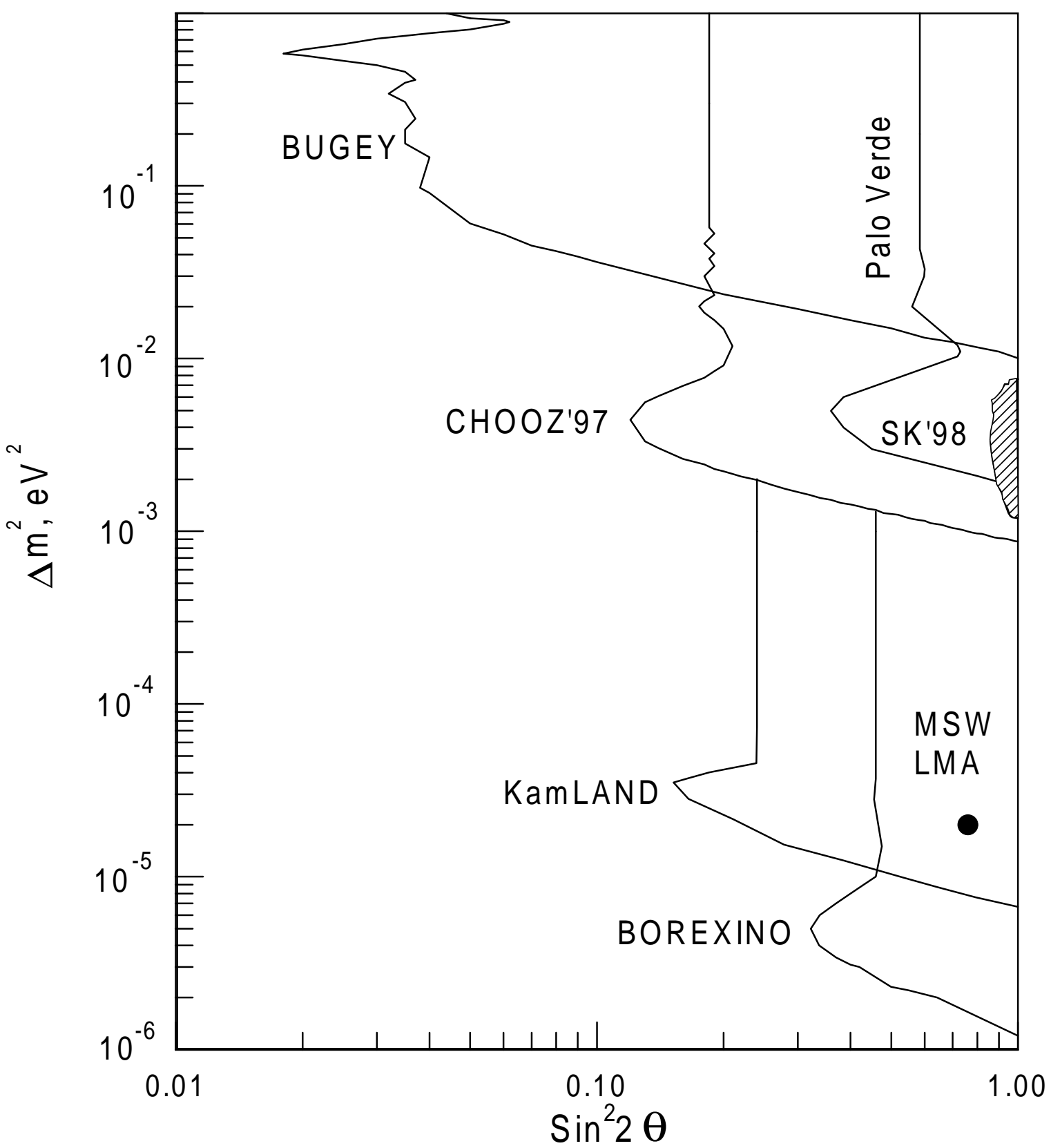

Figure 3. Reactor oscillation param eter plots.

"Bugey" [10], "CHOOZ'97" [1] and "Palo Verde" [11] are $90 \% \mathrm{CL}$ antineutrino disappearence limits; "KamLAND" [6] and "BOREXINO" [7] - are expected $\tilde{v}_{e}$ disappearence sensitivities; "SK'98" [2] is allowed $v_{\mu} \rightarrow v_{\tau}$ oscillation region; "MSW LMA" [5] is the solar $v_{e}$ solution. 


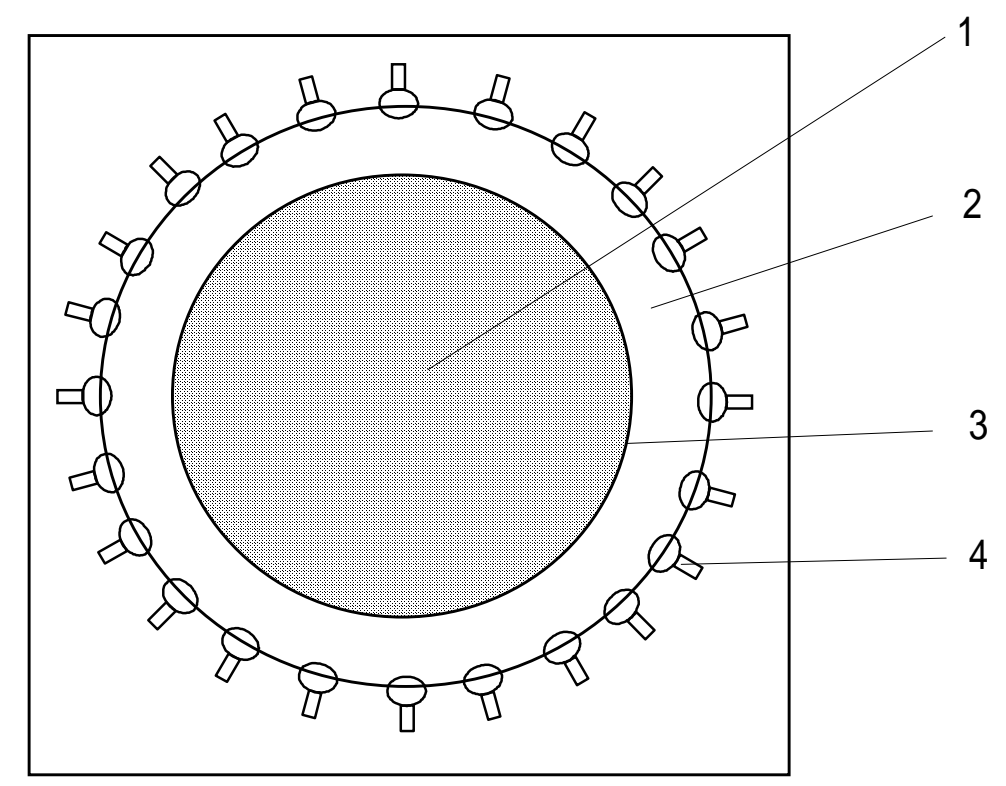

Figure 4. The detector (schematic).

1 - The neutrino target (50 ton mineral oil + PPO).

2 - Mineral oil.

3 - The transparent film.

4 - The PMTs. 


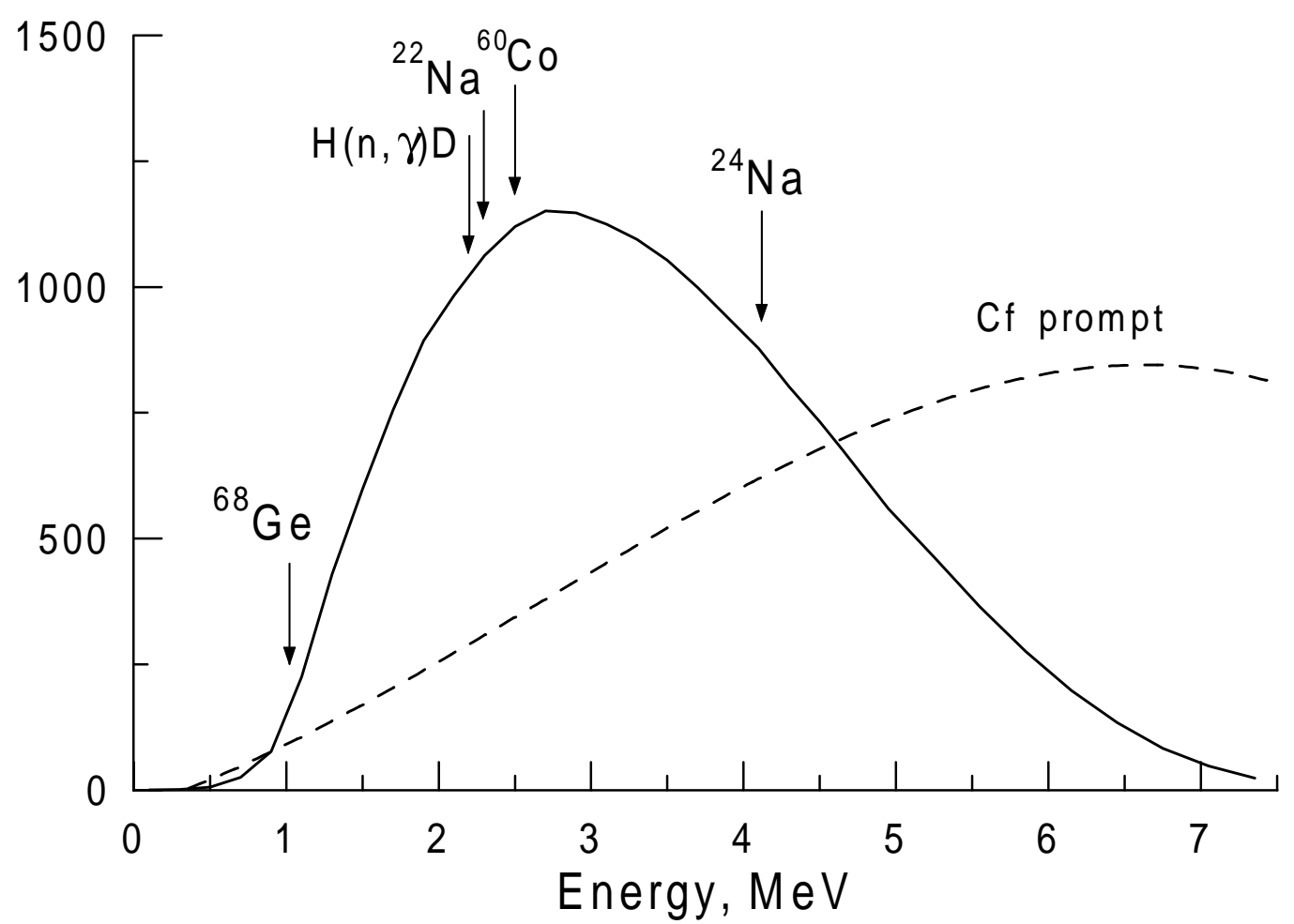

Figure 5. Sources for detector calibrations.

The solid line is the positron energy spectrum. 


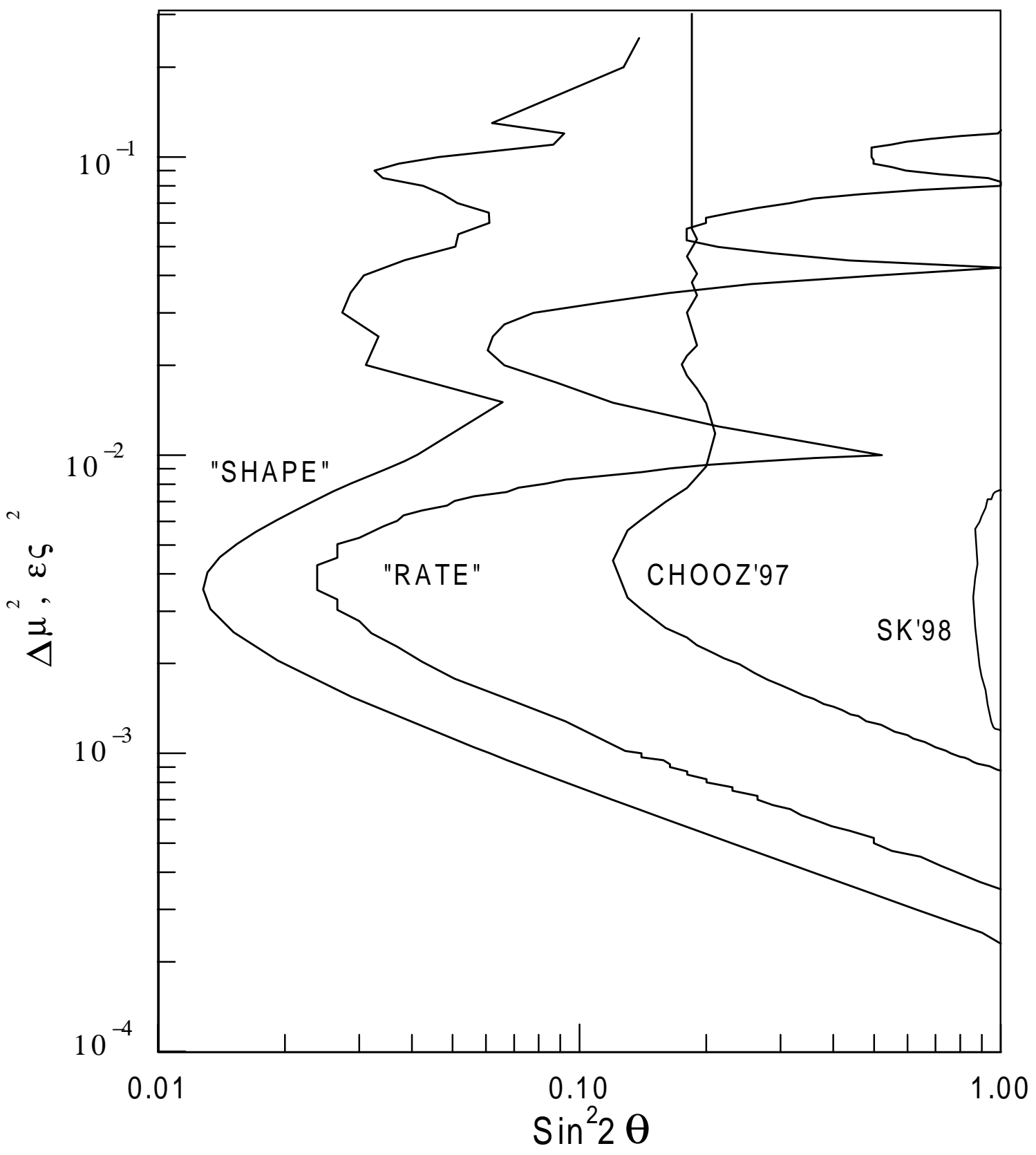

Figure 6. Expected $90 \%$ CL oscillation parameter limits. "RATE" - analysis I, "SHAPE" - analysis II. 